\title{
A LEAD SYSTEM RECORDING TOTAL OUTWARD CARDIAC DIPOLE STRENGTH
}

\author{
BY \\ M. R. BARBER, AND E. J. FISCHMANN \\ From the Naval Research Laboratory, and the Cardiology Department, Green Lane Hospital, Auckland, New Zealand
}

Received January 12, 1961

Unipolar chest leads are said to be selectively sensitive to local potential variation, while limb leads record the heart's total electromotive force without pronounced regional preference. This division did not seem tenable in experiments on leads I, V2, and V5, using torso models of varying shape with hearts of variable size and position (Fischmann and Barber, 1961). In most of the experiments each of the three leads showed unequal sensitivity to different cardiac areas, amounting in some instances to a strong local bias. The bias however depended as much on the characteristics of the model as on the leads, and in some models was as pronounced in lead I as in the "unipolar" leads, whereas in other models, V2and V5 did not show the expected local bias. Since each of the three leads recorded mixtures of unpredictably weighted contributions from various cardiac regions, there was no meaningful and calculable quantitative relationship between a lead and the heart's forces as a whole or in part.

Three orthogonal leads, each consisting of a pair of standard electrodes, are equivalent to but not better than the 12-lead conventional electrocardiogram in the diagnosis of hypertrophy (Threthewie, 1958; Fischmann, 1958; Jacono and Luisada, 1959). They are less satisfactory than conventional leads in the localized changes that accompany infarction (Abildskov et al., 1958; Lancaster et al., 1960). In model studies simple bipolar orthogonal leads, including the most widely used VCG lead systems, share the aforementioned deficiencies of standard lead I (Fischmann and Barber, 1961).

"Aimed electrocardiography," a method that records dipole contributions from strictly limited cardiac regions and excludes contributions from other areas, has been described (Barber and Fischmann, 1961).

The lead field concept of McFee and Johnston $(1953,1954 a, b)$, based on the reciprocity theorem of Green (1828) and Helmholtz (1853), is an important advance toward leads which record sums of uniform contributions from all parts of the heart but the lead system proposed by Johnston's group (Reynolds et al., 1956) does not fully exploit the possibilities of the concept. It follows from the concept that if current enters a torso model through the electrodes of a lead with uniform response, the equipotentials within the model will be straight, equidistant, parallel lines, perpendicular to the axis of the lead. It will be shown that in the horizontal plane, the equipotential fields of the Johnston sagittal and transverse leads deviate from this pattern, whilst in the frontal and sagittal planes the fields, especially that of the transverse lead, are uniform within a heart of normal size but not outside it. Brody (1957) pointed out that "wrapping" a multiple electrode such as that employed in the sagittal lead of Johnston, results in a curved lead field (Fig. 1A).

A new lead-system, also based on the McFee-Johnston lead field concept, is proposed. It includes an electrode which overcomes the Brody slope-effect (Fig. 1B). It has uniform lead fields 

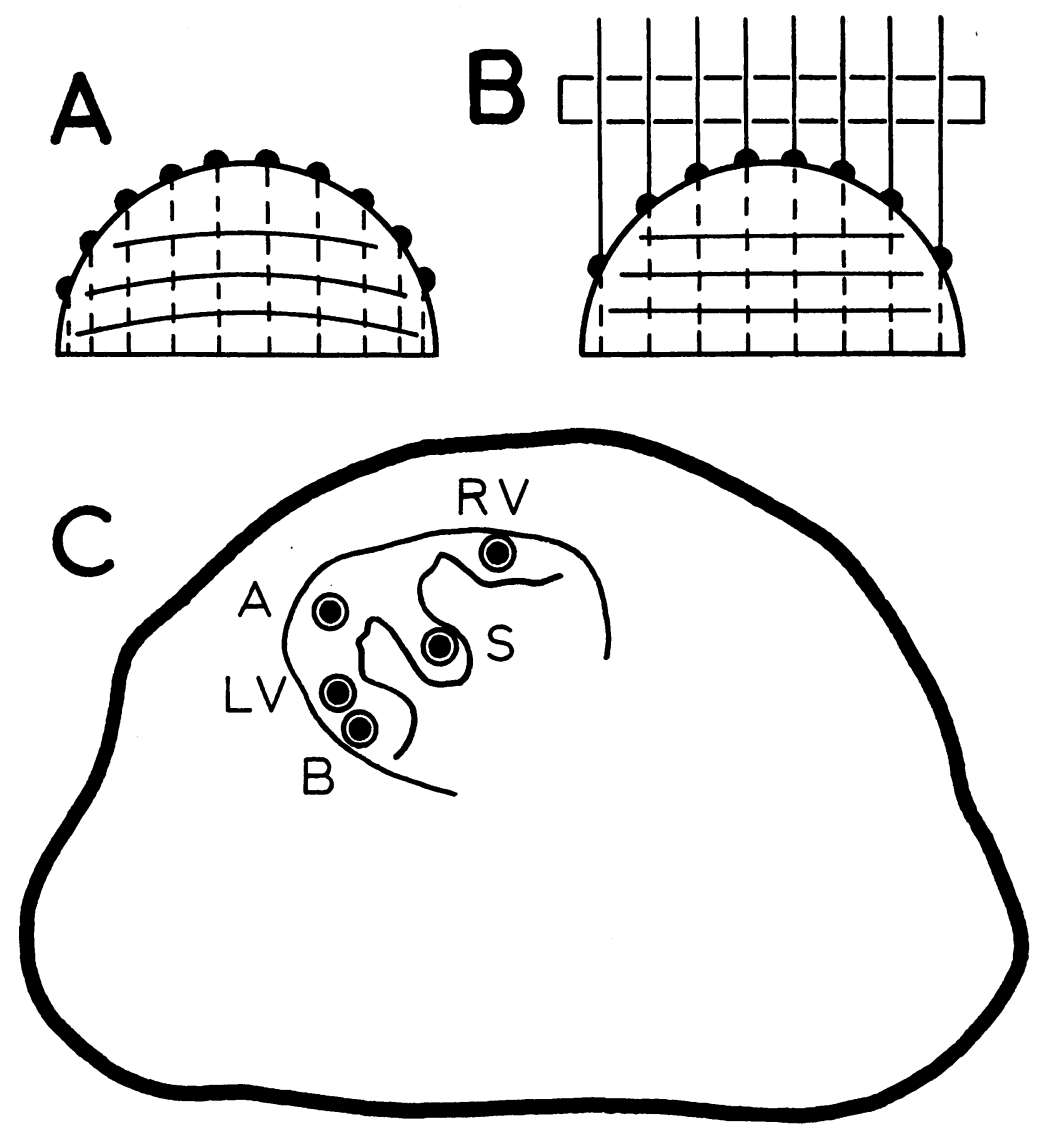

FIG. 1.-(A) "Wrapped" multiple electrode. Projection of electrode units on transverse axis crowded laterally. In equipotential plots this results in a curved field. (B) Slope effect eliminated by platform mounting. (C) Horizontal torso and heart contour, showing 5 arbitrarily selected intramural dipole positions. Torso 1, shown here and in most of the illustrations, and Torso 2 shown only in Fig. 3D, are taken from the cadaver cuts in Fig. 4 and 2 of Walmsley (1958), by courtesy of the author and the editors of the British Heart Journal.

in three planes. The fields are uniform and continuous not only within the heart, but also throughout the chest to the torso surface, and are therefore calculable. The proposed leads will supply the heart's total outward effective dipole moment, a parameter discussed by Gabor and Nelson (1954) but not hitherto clinically applied.

\section{The Proposed Lead System (Fig. 2)}

As no paste is required, leads $\mathrm{X}, \mathrm{Y}$, and $\mathrm{Z}$ take as long to record, as three standard leads. The anterior electrode of the sagittal lead $Z$ (Fig. 2A) consists of $30(6 \times 5)$, the posterior electrode (Fig. 2B) of $20(5 \times 4)$, small balsa wood units, impregnated under vacuum with lithium chloride (Fischmann et al., 1961). The outer rows of each electrode enclose an oblong 14 by 10 in. The electrodes are placed astride the midsternal and vertebral lines respectively, their long sides parallel with these lines. The two electrodes of the transverse lead $\mathrm{X}$ are mounted on a transverse bar. Each of these two electrodes consists of $9(3 \times 3)$ balsa units, the outer rows enclosing 7 by 6 in. 

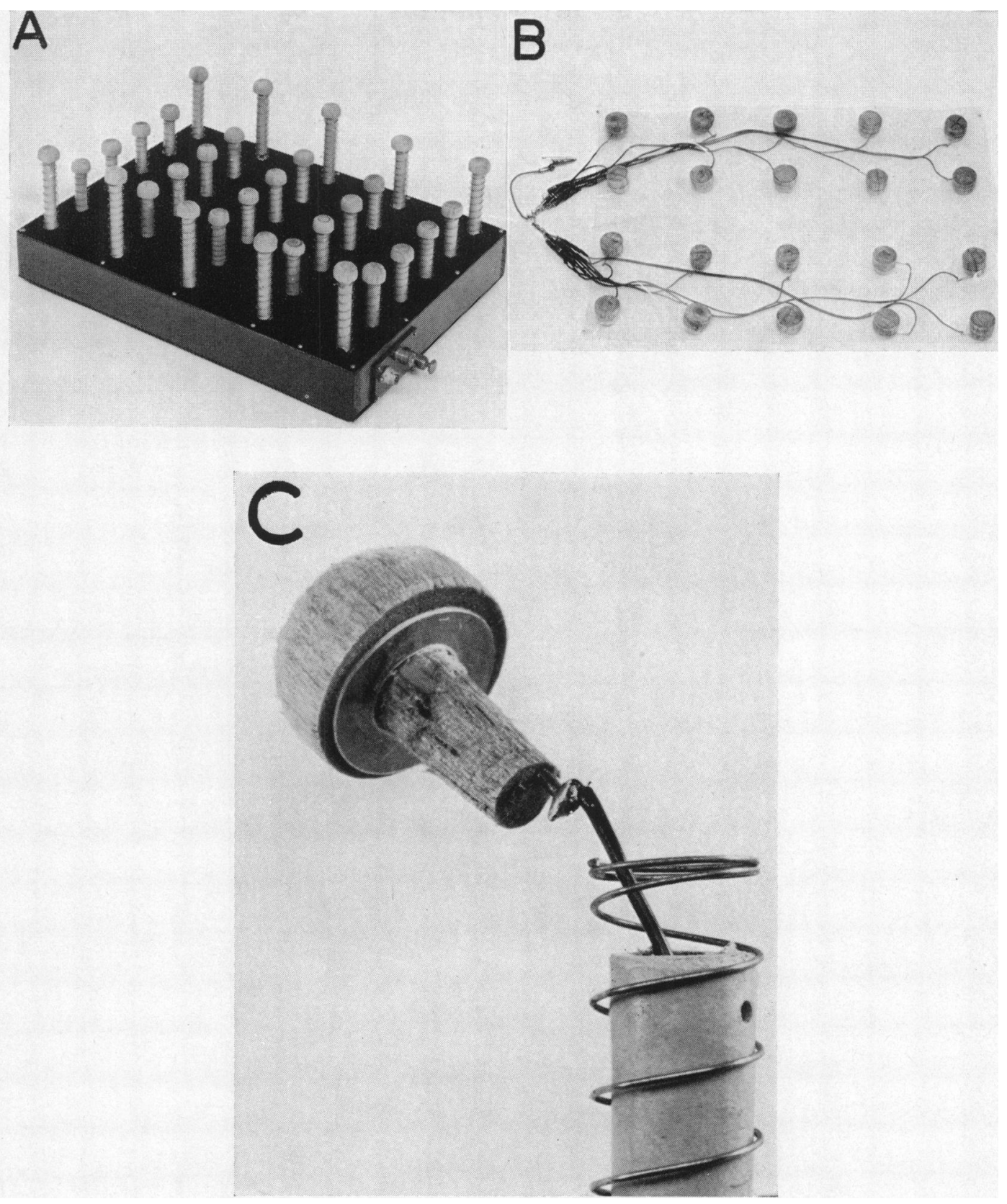

Fig. 2.-The proposed sagittal lead: (A) Platform-mounted anterior electrode, (B) sheet-mounted posterior electrode.

(C) Balsa-lithium "mushroom."

oblongs, each placed astride a midaxillary line, the long sides parallel to that line. Each of the two transverse lead electrodes is connected to a balsa unit placed laterally on the neck.

Two methods of mounting the balsa units were employed: For the dorsal electrode, one in. diameter, grooved balsa discs were "buttoned" into slightly smaller circular holes in a transparent plastic sheet (Fig. 2B). The anterior and the two lateral electrodes consisted of platforms formed by 
two perspex sheets and a wooden frame, penetrated at right angles by arrays of rigid plastic tubes. Small balsa "mushrooms" (Fig. 2C) were forced into the lumina of the tubes. The tubes were spring-loaded so that the "mushrooms" were held away from the platform but sufficient vertical movement was allowed to make contact possible regardless of body shape. When placed on the body, the electrodes are spaced as in Fig. 1B. It can easily be shown that this is equivalent to a uniform peripheral spacing multiplied by the cosine of the slope at any point. A wire was attached to each balsa unit by a stainless steel pin and led away through $56,000 \mathrm{ohm}$ resistors to a common terminal. The vertical lead Y was a head to foot lead (White and Burwell, 1921). Balsa-lithium blocks were interposed between skin and electrode instead of paste (Fischmann et al., 1961).

\section{Two-dimensional (2-D) Lead Field Analysis}

The lead fields of the proposed lead-system are compared with those of other leads developed to record the transverse and sagittal components of the heart's total electromotive force. Several important orthogonal lead systems do not lend themselves to two-dimensional analysis at the heart level, and were not included.

Method. Two horizontal torso outlines, to be called Torso 1 and Torso 2 borrowed from Walmsley's (1958) cadaver cuts, with the kind permission of the author and the editors of this Journal, were scaled to have identical transverse diameters. The heart of Torso 1 (Fig. 1C) was used in both. The pectus excavatum in Fig. 5 was taken from a plaster cast of the living subject. Lead-equipotentials were plotted on Teledeltos paper (Brody and Romans, 1953), cut to torso shape. The complex and irrelevant field details in the immediate vicinity of the electrodes were ignored At five arbitrarily selected points within the heart, dipoles of unit strength were assumed to be present
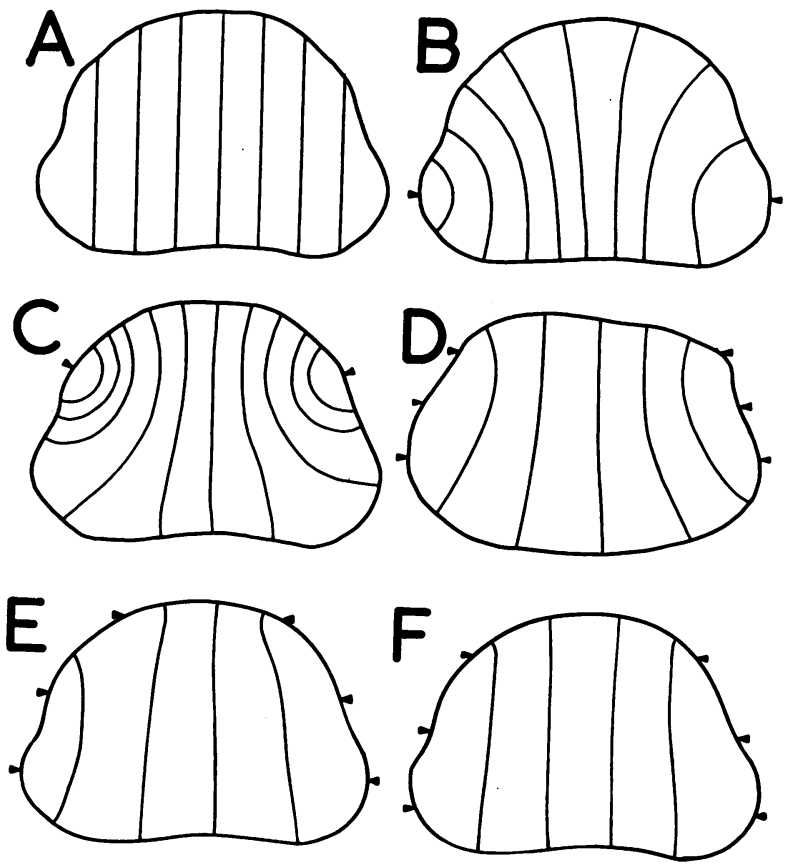

FIG. 3.-Horizontal plane equipotentials of "transverse" leads: (A) Ideally uniform transverse, (B) McFee-Johnston, (C) Simple bipolar, (D and E) Multiple electrodes centered on anterior axillary line, (F) Proposed transverse lead.

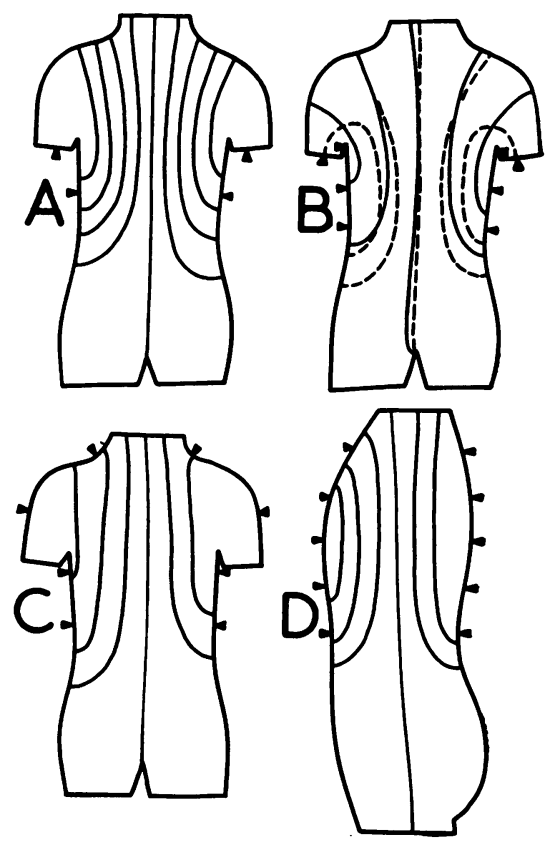

Fig. 4.-(A) McFee-Johnston X. (B) Interrupted lines, field of proposed transverse lead without arm electrodes; solid lines, with added arm electrodes. (C) Proposed transverse lead with bilateral neck electrodes. (D) Proposed sagittal lead, sagittal plane field. 
(Fig. 1C). The dipoles had $\mathbf{X}$ or $\mathbf{Z}$ directions depending on whether lead $\mathbf{X}$ or $\mathbf{Z}$ was studied. To study the effect of heart size, another set of dipoles was obtained by extending by one-half, the straight lines connecting $\mathbf{S}$ with each of the other dipoles. Table I shows the relative strength of the dipoles as recorded by each lead, calculated from the field plots, taking the recorded value of dipole $S$ in each lead as unity. Table II shows relative values for dipole $S$ in each lead, taking the $S$ dipole recorded by a lead with a perfectly uniform field, as unity.

Limitations of 2-D Models. A 2-D field-plot provides precise information about a 3-D body, if both body and electrode cross sections are identical for an infinite distance above and below the cut investigated. Using the McFee-Johnston sagittal lead, or our proposed sagittal and transverse leads, this condition is approximated in horizontal human torso sections at the heart level, less well in sagittal, not at all in frontal plane sections. It can be said however that if a lead is inadequate in a 2-D field plot it will be so in 3-D, but a lead which appears satisfactory in 2-D must yet be shown to be so in 3-D.

Conductivity Distribution Within the Models. The resistivity of lung tissue was considered infinite by Katz et al. (1936), four times that of the rest of the body by Burger and van Milaan (1943) and approaching that of the rest of the body by Schwann and Kay (1957). In view of the contradictory data concerning the largest organ in contact with the heart, it is felt that models having an elaborate conductivity pattern are as likely to be in error as homogeneous models. The high relative conductivity of the heart itself appears established beyond doubt (Burger and van Milaan, 1943; Schwann and Kay, 1957) but as this organ is of finite extent up and downward, a high conductivity cardiac area, whilst desirable in 3-D models (Nelson et al., 1959) is of doubtful value in 2-D plots.

Leads Designed to Record the Transverse Component X (Fig. 3 and Tables I and II). The equipotentials of the perfect transverse lead are equidistant, parallel, straight sagittal lines and all dipoles

TABLE I

Measured Voltages due to Unit Intramural Dipoles, taking the output of Dipole S as UNITy in each model

\begin{tabular}{|c|c|c|c|c|c|c|c|c|}
\hline \multicolumn{2}{|l|}{ Lead } & Torso & Heart & $\mathbf{S}$ & $\mathbf{R V}$ & $\mathbf{A}$ & $\mathbf{L V}$ & $\mathbf{B}$ \\
\hline $\begin{array}{l}\text { Johnston (Fig. 3B) } \\
\text { Simple bipolar (Fig. 3C) }\end{array}$ & $\mathbf{X}$ & $\begin{array}{l}1 \\
2 \\
1 \\
1 \\
2 \\
1 \\
1\end{array}$ & $\begin{array}{l}\mathbf{N} \\
\mathbf{N} \\
\mathbf{L} \\
\mathbf{N} \\
\mathbf{N} \\
\mathbf{L} \\
\mathbf{N}\end{array}$ & $\begin{array}{l}1.00 \\
1.00\end{array}$ & $\begin{array}{l}0 \cdot 80 \\
0 \cdot 85 \\
0 \cdot 69 \\
1.0 \\
1.0 \\
0.84\end{array}$ & $\begin{array}{l}0.85 \\
0.99 \\
0.71 \\
1.0 \\
1 \cdot 15 \\
1.44\end{array}$ & $\begin{array}{l}1.08 \\
1.13 \\
0.98 \\
0.90 \\
0.96 \\
1.44\end{array}$ & $\begin{array}{l}1 \cdot 19 \\
1 \cdot 30 \\
1 \cdot 32 \\
0.84 \\
0.91 \\
0.72\end{array}$ \\
\hline Proposed (Fig. 3F) & & $\begin{array}{l}1 \\
2 \\
1\end{array}$ & $\left.\begin{array}{l}\mathbf{N} \\
\mathbf{L}\end{array}\right\}$ & 1.00 & 1.00 & 1.00 & 1.00 & 1.00 \\
\hline Johnston (Fig. 5) & $\mathbf{Z}$ & $\begin{array}{l}1 \\
2 \\
1\end{array}$ & $\begin{array}{l}\mathbf{N} \\
\mathbf{N} \\
\mathbf{L}\end{array}$ & 1.00 & $\begin{array}{l}0.86 \\
0.93 \\
0.68\end{array}$ & $\begin{array}{l}1 \cdot 18 \\
1 \cdot 50 \\
1 \cdot 34\end{array}$ & $\begin{array}{l}1.13 \\
1.26 \\
1.42\end{array}$ & $\begin{array}{l}1.20 \\
1.22 \\
1.31\end{array}$ \\
\hline Simple bipolar (Fig. 5) & $\mathbf{Z}$ & $\begin{array}{l}1 \\
2 \\
1\end{array}$ & $\begin{array}{l}\mathbf{N} \\
\mathbf{N} \\
\mathbf{L}\end{array}$ & 1.00 & $\begin{array}{l}0.79 \\
1.07 \\
0.50\end{array}$ & $\begin{array}{l}0.94 \\
1.04 \\
0.73\end{array}$ & $\begin{array}{l}0.76 \\
1.02 \\
0.71\end{array}$ & $\begin{array}{l}0.83 \\
1.02 \\
0.80\end{array}$ \\
\hline Unipolar lead (Fig. 5) & $\mathrm{VB}^{*}$ & $\begin{array}{l}1 \\
1 \\
2\end{array}$ & $\underset{\mathbf{N}}{\mathbf{N}}$ & 1.00 & $\begin{array}{l}0.24 \\
0.61\end{array}$ & $\begin{array}{l}0.77 \\
1.00\end{array}$ & $\begin{array}{l}1.07 \\
1.20\end{array}$ & $\begin{array}{l}1 \cdot 21 \\
1 \cdot 20\end{array}$ \\
\hline Proposed (Fig. 5) & $\mathbf{Z}$ & $\begin{array}{l}1 \\
2 \\
1\end{array}$ & $\left.\begin{array}{l}\mathbf{N} \\
\mathbf{N} \\
\mathbf{L}\end{array}\right\}$ & 1.00 & 1.00 & 1.00 & 1.00 & 1.00 \\
\hline
\end{tabular}

S, RV, A, LV, and B are intramural points as in Fig. 1C. Another set of four points, representing an "enlarged" heart was obtained by adding 50 per cent to the straight lines connecting $S$ with each of the other 4 points. N, normal; L, "enlarged" heart. Measurements are subject to the limitations imposed by 2-D models, as discussed in text. Torso 1 and Torso 2: see legend of Fig. 1C.

*VB: lead $\mathrm{Z}$ of the tetrahedron lead system.

$2 \mathrm{U}$ 
TABLE II

Relative Values for Dipole S (Fig. 1C) in each lead, taking the S Dipole Recorded by a lead with Ideally UNIFORM FIELD, AS UNITY

\begin{tabular}{ll|c|c}
\hline \multicolumn{1}{c|}{ Lead } & & Torso 1 & Torso 2 \\
\hline Johnston (Fig. 3B) & $\mathrm{X}$ & 0.82 & 1.06 \\
Simple bipolar (Fig. 3C) & $\mathrm{X}$ & 1.40 & 1.64 \\
Proposed (Fig. 3F) & $\mathrm{X}$ & 1.00 & 1.00 \\
\hline Johnstion (Fig. 5) & $\mathrm{Z}$ & 1.10 & 0.93 \\
Simple bipolar (Fig. 5) & $\mathrm{Z}$ & 1.75 & 1.50 \\
Unipolar lead (Fig. 5) & VB & 0.12 & 0.22 \\
Proposed (Fig. 5) & $\mathrm{Z}$ & 1.00 & 1.00 \\
\hline
\end{tabular}

X's to be kept separate from Z's, since the proposed lead X gives a higher voltage output than the proposed Z, by a factor 1.33. Measurements are subject to the limitations imposed by $2-\mathrm{D}$ models, as discussed in text. Torso 1 and Torso 2: see legend of Fig. 1C. VB: lead $\mathrm{Z}$ of the tetrahedron lead system.

have a recorded magnitude of $1 \cdot 0$. The equipotentials of the McFee-Johnston X leads fan out toward the front. The field is more uniform behind than within the heart, with decreased sensitivity to anterior dipoles. A marked difference between RV and the posterior dipoles, the difference increasing with heart size, is seen in Tables I and II. A transverse bipolar chest lead with anatomical axis through the heart (White and Burwell, 1921; Wenger and Hupka, 1956) gives a more uniform intracardiac field but considerable inter-dipole variation still occurs with the larger heart. Three electrodes placed astride the anterior axillary lines, with two kinds of spacing, have fields similar to that of the bipolar chest lead.

A highly uniform field is achieved by spacing three electrodes 3 in. apart and placing them astride the midaxillary lines. Fig. 4 shows that the frontal plane field of this arrangement is further improved by an electrode placed laterally on the neck.

Leads Designed to Record the Sagittal Component Z (Fig. 5 and Tables I and II). The equipotentials of the ideal sagittal lead are equidistant, parallel, straight transverse lines and all dipoles have recorded magnitudes of $1 \cdot 0$. The field of the Johnston $\mathrm{Z}$ lead shows convergence of equipotentials toward the left. The calculated dipole magnitude shows variation within each of the two torsos. Sensitivity of the lead, to torso shape and heart size, is suggested by the difference in the extent of inter-dipole variation in the three sets shown for this lead in Table I and the difference between the $\mathrm{S}$ dipoles of torsos 1 and 2 (Table II). A single electrode in front and behind the heart (White and Burwell, 1921; Wenger and Hupka, 1956; Jacono and Luisada, 1959) gives a more uniform field in the horizontal plane than does the sagittal McFee-Johnston lead. Lead VB, used to supply the $\mathrm{Z}$ component of the tetrahedron lead-system, has a highly inadequate field. It records an unpredictable mixture of $\mathrm{Z}$ and $\mathrm{X}$ dipole components. The field strength within the heart itself is so small as to render the calculation of dipole strength difficult. The values shown for VB in the tables are therefore approximate. They suggest highly unequal sensitivity to dipoles throughout the heart.

The horizontal 2-D lead-field of the proposed sagittal lead is uniform in torsos 1 and 2, in the presence of breasts, and in a torso with pectus excavatum. It approximates the ideal sagittal lead, regardless of chest shape (Fig. 5).

The Vertical Component Y. A head-to-foot lead was used. It is seen in Fig. 6 that the field of this lead is only approximately uniform. A Y lead with a more satisfactory field is being developed.

Quantitative approach to Electrocardiography. The proposed lead-system was designed to fulfil two essential conditions of a quantitative relationship between the heart's total force and the ECG: First, lead fields must be uniform and calculable throughout the body to the body surface. Lead systems with uniform fields within the heart but not throughout the torso, record voltages which are proportional to the dipole moment but do not give the quantitative value of the latter. Second, as far as practicable, electrodes must cover all areas, where $\mathbf{X}$ and $\mathbf{Z}$ components respectively are 

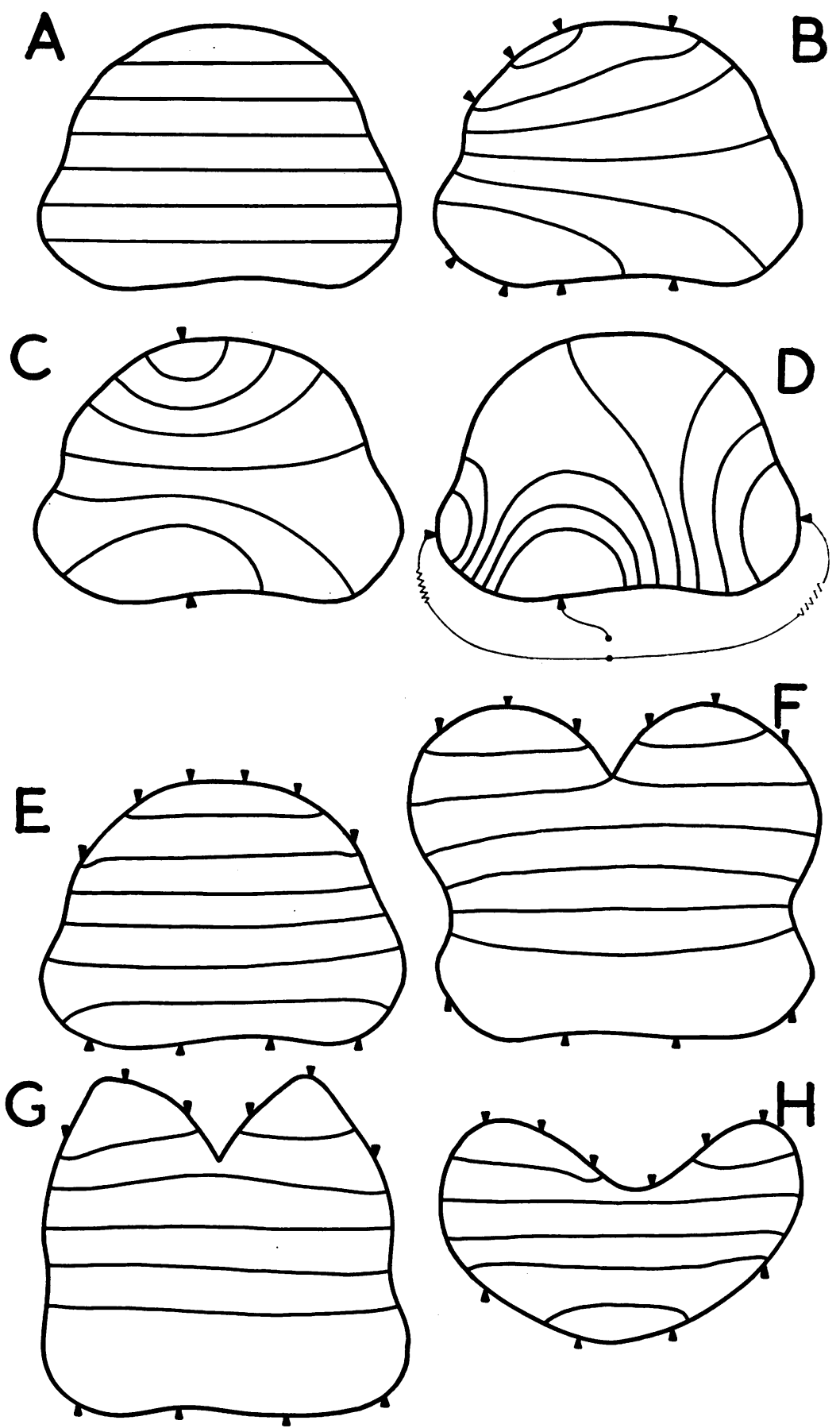

Fig. 5.-Horizontal plane equipotentials of "sagittal" leads: (A) Ideally uniform sagittal, (B) McFee-Johnston sagittal, (C) Simple bipolar, (D) Sagittal lead of tetrahedron. (E-H) Proposed sagittal lead, (E) Torso I, (F, G) Breasts added to Torso I, (H) Pectus excavatum from plaster cast of patient. 
present. They must therefore extend as far as possible in all directions beyond the region of the heart.

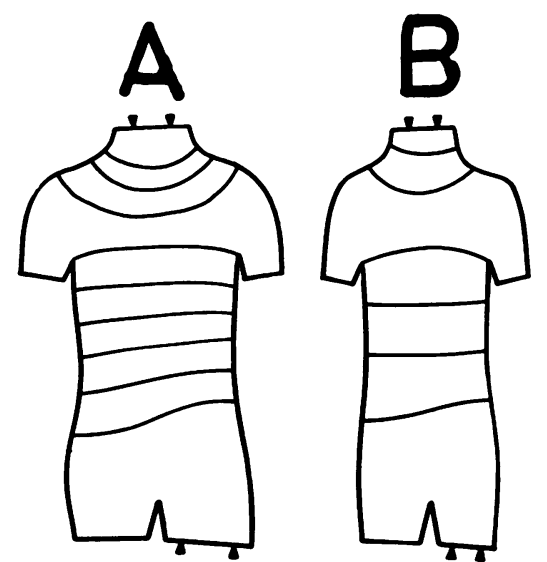

Fig. 6.- "Vertical" lead. (A) Thickset and (B) slender torso.

The Total Dipole Moment of the Heart. A current dipole is a doublet consisting of a source of current I amps and an equal current sink situated a short distance "d cm." away. The dipole moment $\mathbf{M}$ is defined as the product (I.d.). By integrating over the body surface (Gabor and Nelson, 1954), i.e. summing the individual voltagearea (V.A.) contributions of each unit of a multiple electrode, the heart's resultant total outward directed dipole moment is obtained. The $\mathrm{X}$ and $\mathrm{Z}$ components of the moment are given by the formulæ

$$
\mathrm{Mx}=\frac{\text { (V.Ayz) }}{\rho} \quad \mathrm{Mz}=\frac{(\mathrm{V} . \mathrm{Axy})}{\rho}
$$

$\mathrm{Mx}$ and $\mathrm{Mz}$ are the total dipole moments in the $\mathrm{X}$ and $\mathrm{Z}$ directions, Ayz and Axy areas of the left and anterior electrode banks respectively and $\rho$ the mean resistivity of the body. $V$ is the voltage measured between the pairs of the electrode banks in each case. The right and dorsal electrodes deduct right and backward $\mathrm{X}$ and $\mathrm{Z}$ components.

The area $\mathrm{A}$ of a multiple electrode is the number of balsa units multiplied by a small square area enclosing the unit and extending half a unit space in 4 directions. This is equivalent to an area enclosed by a rectangle lying half a space outside the cluster. It applies precisely if the squares are infinitely small or the fields within them uniform. Correction for any single unit not in contact with the skin is impossible, as its voltage contribution is unknown. A complete marginal row not in contact, systematically decreases the electrode area and correction is possible. Voltages outside area $A$ have been neglected. The error due to this has been estimated in teledeltos models and found to be no greater than 10 per cent.

Mean body resistivity is taken as 480 ohms. cm., Burger and van Milaan (1953) and Schmitt (1957) having observed values of 450 and 489 respectively.

The $\mathrm{Y}$ component of the heart's dipole moment is given by the fomula $\mathrm{My}=\frac{\mathrm{V} \text {.At }}{\rho}$ where $\mathrm{V}$ is the voltage of the head to foot lead $\mathrm{Y}$ at the transverse torso area at heart level. The field plots in Fig. 6 suggest that torso shape affects the voltage of lead $Y$ : field strength is inversely proportional to the cross-sectional area of the torso, that is, other things being equal, the $\mathrm{Y}$ voltage is higher in slender than stocky subjects.

Substituting the values for mean resistivity and electrode area in the three formulæ, the $\mathrm{X}, \mathrm{Z}$, and $\mathrm{Y}$ components of the heart's total dipole force (in volt. $\mathrm{cm}$. units) are obtained by multiplying the voltages of leads $\mathrm{X}, \mathrm{Z}$, and $\mathrm{Y}$ by $1 \cdot 27,2 \cdot 82$ and $\frac{\mathrm{At}}{480}$ respectively. A multiplying factor of $2 \cdot 25$ must be used if one horizontal, of $2 \cdot 35$ if one vertical, row of the sagittal lead electrode is not in contact with the skin. The multiplying factors for the transverse lead electrode are $\mathbf{0 . 8 5}$ for a missing horizontal, 0.85 for a vertical row. The figures for the transverse electrode are approximate owing to the limited area covered. The lead field tends to fan out from an electrode of limited size and is therefore smaller in magnitude than that computed from the electrode area. The teledeltos model error is generally not more than 10 per cent, because the field is constrained by the body surface.

Closed Irregular Dipole Surface (Green Surface) as alternative to single Equivalent Dipole. Traditionally the heart's electromotive forces are often treated as a single summation dipole. It has never been assumed that such a dipole actually exists anywhere within the heart. The evidence showing 
that even a hypothetical single dipole is not wholly tenable was reviewed and extended by McFee and Paraungao (1959).

The resistivity of blood is several times lower than that of the rest of the body (Burger and van Milaan, 1943; Schwann and Kay, 1957). The heart is thus an accumulation of dipoles in and around a time variable and irregularly shaped high conductivity area within a body of lower conductivity. As a working hypothesis, to define the relationship between the heart's forces and body surface potentials recorded by satisfactory leads, it is possible to replace the heart by a closed dipole surface known to physicists as a Green surface (Green, 1828; Smythe, 1950), having the following properties: first the surface is perpendicular at all points to the equipotentials of the heart's electric field; second, all dipoles on the surface act normally to it; thirdly the resultant dipole moment per unit area at any point on the surface is numerically equal to the potential at that point divided by the resistivity of the medium.

There is an infinite number of Green surfaces between the high conductivity cardiac area and the surface of the body. The outward electrical effects of each of these surfaces are identical. Two are of particular interest, "the smallest Green surface" directly bounding the heart's high conductivity area, and the surface of the body itself. As the two produce the same outward effect, it follows that dipole integration on the body surface is in fact integration on the "smallest Green surface." Likewise any lead-system which computes orthogonal components from the body surface, produces the components of the dipole force on the "smallest Green surface," i.e. on the surface of the heart's high conductivity area.

\section{SUMMARY}

The McFee-Johnston lead field concept is an important advance toward electrocardiographic leads giving uniform and calculable dipole representation throughout the heart but the $\mathrm{X}$ and $\mathrm{Z}$ leads suggested by Johnston's group do not take full advantage of the concept. The equipotential fields of these leads are non-uniform in horizontal plane models, while in the frontal and sagittal planes, field uniformity does not extend to the torso surface. The fields also suffer from distortion by the Brody slope effect, as a result of "wrapping" multiple electrodes.

A new lead system, also based on the McFee-Johnston concept, is proposed. It includes a platform-mounted multiple electrode, which automatically corrects for the slope effect. In 2-D field plots the $\mathrm{X}$ and $\mathrm{Z}$ fields are uniform in three planes, the leads being thus insensitive to dipole location and distribution, regardless of torso shape and heart size. Since the fields are uniform and calculable, not only in the heart but throughout the torso to the torso surface, a calculable quantitative relationship exists between cardiac and surface forces. A method of determining the heart's total outward directed dipole moment is therefore described.

A closed irregular dipole surface (Green surface) at the boundary of the high conductivity area formed by intracardiac blood, is suggested as an alternative to the widely used but inaccurate single equivalent cardiac dipole.

The limitations of 2-D models, especially in the frontal and sagittal planes, are stressed.

The six electrodes of the three proposed leads consist of a total of 72 balsa-lithium units. As no paste is required, the three leads take no longer to record, than 3 standard leads using paste.

\section{REFERENCES}

Abildskov, J. A., Street, W. W., Solomon, N., and Toomajian, A. H. (1958). Circulation, $12,1069$.

Barber, M. R., and Fischmann, E. J. (1961). Nature. To be published.

Brody, D. A. (1957). Amer. Heart J., 53, 174.

- and Romans, W. E. (1953). Amer. Heart J., 45, 263.

Burger, H. C., and van Milaan, J. B. (1943). Acta Med. Scand., 114, 584.

Fischmann, E. J. (1958). Brit. Heart J., 20, 167.

- Seelye, R., and Crutcher, R. (1961). To be published.

Gabor, D., and Nelson, C. V. (1954). J. Appl. Physics, 25, 413. 
Green, G. (1828). An Essay on the Application of Mathematical Analysis to the Theory of Electricity and Magnetism. Nottingham. Quoted from Mathematical Papers of the late G. Green. (1871). Macmillan, London.

Helmholtz, H. (1853). Ann. Physiol. u. Chem., 29, 222.

Jacono, A., and Luisada, A. A. (1959). Amer. J. Cardiol., 4, 218.

Katz, L. N., Sigman, E., Gutman, I., and Ocko, F. H. (1936). Amer. J. Physiol., 116, 343.

Lancaster, W. M., Semple, T., and Kelly, J. C. C. (1960). Brit. Heart J., 22, 347.

McFee, R., and Johnston, F. D. (1953). Circulation, 8, 554.

$\longrightarrow$, (1954a). Circulation, 9, 255. (1954b). Circulation, 9, 868. , and Paraungao, A. (1959). Amer. Heart J., 58, 582.

Nelson, C. V., Chatterjee, M., and Austin, W. H. (1959). Circulation, 20, 745.

Reynolds, E. W., Jr., Cordes, J. F., Willis, P. W. III, and Johnston F. D. (1956). Circulation, $14,48$.

Schmitt, O. H. (1957). Ann. N. Y. Acad. Sci., 65, 1109.

Schwann, H. P., and Kay, C. F. (1957). Ann. N. Y. Acad. Sci., 65, 1007.

Smythe, W. R. (1950). Static and Dynamic Electricity. p. 57. McGraw Hill, New York.

Trethewie, E. R. (1958). Brit. med. J., 2, 1428.

Walmsley, Robert (1958). Brit. Heart J., 20, 441.

Wenger, R., and Hupka, K. (1956). Klinische Vektorkardiographie. Steinkopff, Darmstadt.

White, P. D., and Burwell, C. S. (1921). Proc. Amer. Soc. Clin. Invest., Amer. Med. Assoc. Press. 